\title{
Does high fat diet effect the bone-implant connection?
}

\author{
Dundar $\mathrm{S}^{1}$, Bozoglan $\mathrm{A}^{1}$, Sahin $\mathrm{K}^{2}$, Balci $\mathrm{TA}^{3}$, Kirtay $\mathrm{M}^{4}$, Bozoglan $\mathrm{MY}^{5}$, Calisir $\mathrm{M}^{6}$ \\ Firat University, Faculty of Dentistry, Department of Peridontology, Campus, Elazig, Turkey. \\ sdundar@firat.edu.tr
}

\begin{abstract}
OBJECTIVE: Obesity induced by a high fat diet is associated with chronic up-regulation of inflammatory cytokines which stimulate osteoclast activity and bone resorption. However, the role of high-fat diet on boneimplant connectivity has not been studied in detail. In this study, we investigated whether a high-fat diet (HFD) affects bone implant connection (BIC) in periimplant bone.

METHODS: Twenty female Sprague Dawley rats were divided in two groups: 1) Control rats were fed with normal chow and titanium implants were integrated into tibial bones at the end of $3^{\text {rd }}$ month and no treatment was applied 2) HFD group; rats were fed a high-fat diet ( $42 \%$ of calories as fat), then the titanium implants were integrated into tibial bones at the end 3rd month. Following surgical integration of the implants, the rats were fed with control and HFD diets for 3 months. After the 6 months experimental period all rats were sacrificed and the implants and surrounded bone tissues were collected and the BIC was assessed histomorphometrically after the non-decalcifiing histological methods. Bone implant connection was detected with the ratio of the implant surface directly connected with the peri-implant bone tissues to the total implant surface length.

RESULTS: Histologic analysis showed that HFD was not impaired BIC $(p>0.05)$.

CONCLUSION: In conclusion, within the limitation of this research, HFD did not effect the BIC rat tibias (Tab. 2,

Fig. 2, Ref. 26). Text in PDF www.elis.sk.

KEY WORDS: bone-implant contact, osseointegration, dental implants, high fat diet.
\end{abstract}

\section{Introduction}

Dental implant supported prostheses have become a widely accepted and scientifically accepted treatment option for the treatment of edentulousness. However, the systemic condition of the patient, the quality and quantity of the bone to which the dental implant is placed, the geometric design and the surface properties of the implant, are key factors for success in dental implant supported prosthetic treatment $(1,2)$.

The main health problems of the aging population include atherosclerosis-vascular calcification and osteoporosis. In addition to the vascular problems of hyperlipidemia induced by highfat diet (HFD), adverse effects on the cardiovascular system are also known. In hyperlipidemic individuals, lipoproteins migrate through the endothelium into the internal subendothelium. These

${ }^{1}$ Firat University, Faculty of Dentistry, Department of Periodontology, Elazig, Turkey, ${ }^{2}$ Firat University, Faculty of Veterinary Medicine, Department of Animal Nutrition and Nutritional Diseases, Elazig, Turkey, ${ }^{3}$ Firat University, Faculty of Medicine, Department of Nucleer Medicine, Elazig, Turkey, ${ }^{4}$ Oral and Maxillofacial Surgeon, Private Practice, Toronto, Canada, ${ }^{5}$ Ministry of Health, Elazig Health Directorate, Department of Pharmacology, Elazig, Turkey, and ${ }^{6}$ Adiyaman University, Faculty of Dentistry, Department of Periodontology, Adiyaman, Turkey

Address for correspondence: S. Dundar, Dr, Firat University, Faculty of Dentistry, Department of Peridontology, 23119, Campus, Elazig, Turkey. Phone: +90.533 .5819238$ lipoproteins are captured and oxidatively modified by metabolically active adjacent smooth muscle cells and reactive oxygen species produced by macrophages. A similar cycle is seen in the case of osteoporosis induced by hyperlipidemia, while lipid particles bound to the oxidized protein accumulate in the perivascular subendothelial areas. Osteoblast cells have the ability to oxidatively modify lipoproteins and oxidized lipid products were identified in the bone marrow in hyperlipidemic condition (3-6). High-fat diets increase protein-bound lipid particles known as lipoproteins and reactive oxygen species. In addition, lipid-derived reactive oxygen species have been reported to reduce osteoblastic differentiation. Oxidized lipids induce osteoclastogenesis and bone morphogenetic protein-2 release. In vivo studies report that hyperlipidemia produces bone loss. It was reported that HFD may produce deleterious effects on the absorption of dietary calcium and consequently an adverse effect on bone mineralization in animals $(4,6,7)$. Many studies have linked obesity with diabetes and hypercaloric dietinduced obesity to morbidity resulting from periodontal disease $(8,9)$. Additionally, Fujita and Maki. reported that HFD-induced obesity during growth not only triggers mandibular osteoporosis but also increases the risk of spontaneous periodontal disease (9). However, the role of the high-fat diet on bone-implant connection (BIC) has not been studied in detail. Therefore, the aim of this study is to investigate the effects of HFD on BIC of titanium implants in rat tibial bones for three months before surgical application and three months after surgical implantation. 


\section{Material and methods}

Twenty female Sprague Dawley rats (aged 4-6 month; weighing 220-280 gr) were obtained from Experimental Research Center, Firat University, Elazig, Turkey in the same estrus. The animals were housed in temperature-controlled cages, subjected to a 12hour /12-hour light/ dark cycle, and allowed free access to food and water throughout the 6 months experimental period. All the surgical and experimental application were applied at the Firat University Experimental Research Center. The ethic approval of this research was obtained from Firat University Animal Experimental Ethics Council, Elazig, Turkey. The recommendations of the Helsinki Declaration on the protection of experimental animals used in the research were fully complied with. The rats were divided randomly into two groups as follows 1) Control Group: Rats fed with normal chow ( $12 \%$ of calories as fat) throughout the experimental period of 6 months and machined surfaced titanium implants were surgically inserted in right tibias after the 3 months normal feeding and the rats were fed with normal diet again after the surgical integration of the implants for 3 months (10). 2) High-fat diet Group (HFD): Rats fed with high-fat diet [ $42 \%$ of calories as fat] throughout the experimental period of 6 months and titanium implants were integrated into right tibias surgically at the end of $3 \mathrm{rd}$ month (10). And after the surgical application of the machined surfaced titanium implant rats were fed for 3 months with HFD again. After the six months rats were sacrificied and the titanium implants with surrounded bone tissues were removed for histological analysis.

\section{Surgical procedures}

General anesthesia was administered using $35 \mathrm{mg} / \mathrm{kg}$ ketamine hydrochloride and $5 \mathrm{mg} / \mathrm{kg}$ xylazine intramuscularly. After the general anesthesia the tibial bone was washed and shaved before the surgical application. All the surgical applications were done under sterile conditions. The incision of the skin was made on the tibial crest. The periosteal elevator was used for lifting the flap and periost to reach the metaphyseal part of the tibial bone. Implant sockets were created with the surgical drill with saline irrigation to avoiding necrosis of the bone tissues. After the implantation of the titanium implants, the tibial skin was sutured with 4-0 polyglactin resorbable sutures. Antibiotic (40 mg/kg cephalosporin) and analgesic $(0.1 \mathrm{mg} / \mathrm{kg}$ tramadol hydrochloride) were injected intramuscularly for 3 days after the surgical operations. All the surgical applications were done by the same researcher.

\section{Histological analysis}

The specimens were fixed in $10 \%$ formaldehyde solution. After the fixation, the implants and surrounding bone tissue were embedded into a 2-hydroxyetylmetacrylate resin to allow cutting of the non-decalcified bone and titanium with the Exakt microtome (Germany). Each section was ground with the Exakt grinder and a $50 \mu \mathrm{m}$ thickness section was obtained for the light microscope analysis. For the histological staining methylene blue was used. Histological analyses of BIC were then performed using an image analyzer (Nikon, Japan) at the Firat University Faculty of Medicine's Department of Microbiology Laboratory. Three samples were removed from both groups due to improper preparation. In each implant, the level of BIC was detected as the ratio of the implant surface directly touching the bone to the total implant surface length (6).

\section{Biochemical analysis}

Blood samples were taken from rats heart under deep anesthesia. The samples were centrifuged at $3000 / \mathrm{g}$ for $10 \mathrm{~min}$, and the blood serum was collected. The serum samples were stored at $-80{ }^{\circ} \mathrm{C}$ until analyzed. Serum glucose, low density lipoprotein (LDL), very low density lipoprotein (VLDL), triglyceride, aspartate aminotransferase (AST), alanine aminotransferase (ALT), alkaline phosphatase, calcium, phosphorus parameters were analysed by routine biochemical methods obtained from the serum samples of rats at the Department of Biochemistry in the Faculty of Medicine of Firat University.

\section{Dual energy X-ray absorptiometry (DEXA) analysis}

After the mandibular bones were taken out of the rats, dual energy X-ray absorptiometry analysis was pareformed at Firat University, Faculty of Medicine, Department of Nucleer Medicine, Elazig, Turkey (Hologic, Discovery Series, USA). Two samples were not included in the analysis in the ND group due to not being obtained properly. Bone mineral density (BMD) and bone mineral content (BMC) analysis were done with dual energy X-ray absorptiometry analysis.

\section{Statistical analysis}

SPSS software was used for statistical analysis. The mean values \pm standard deviation values of the mean for each group were calculated. A Student's t-test was used in the analysis of data, and $\mathrm{p}$ $<0.05$ were considered sufficient to indicate statistical significance.

\section{Results}

\section{Histological results}

The results of the histomorphometric analysis of the BIC in all groups are shown in Table 1. Statistically, a significant difference was not detected between the ND and the HFD groups for BIC ( $>>0.05$ ) (Figs $1 \mathrm{a}, \mathrm{b}$, and $2 \mathrm{a}, \mathrm{b})$.

\section{Biochemical results}

The results of the biochemical analysis of the glucose, AST, ALT, LDL, VLDL, Ca, P, ALP, DEXA parameters and triglyceride in all groups are shown in Table 2. Statistically, a significant difference was not detected between the ND and the HFD groups for glucose, AST, ALT, Ca, ALP, P levels ( $p>0.05$ ). Additionally, serum LDL $(p=0.002)$, VLDL $(p=0.001)$, and triglyceride

Tab. 1. Bone implant contact of the groups.

\begin{tabular}{lccccc}
\hline Parameter & Groups & $\mathrm{n}$ & Mean & SD & $\mathrm{p}$ \\
\hline \multirow{2}{*}{ BIC } & HFD & 7 & 68.33 & 7.82 & $>0.05$ \\
\cline { 2 - 6 } & ND & 7 & 70.93 & 6.10 & $>0.05$ \\
\hline
\end{tabular}

Student $\mathrm{t}$ test $(\mathrm{p}>0.005)$ 

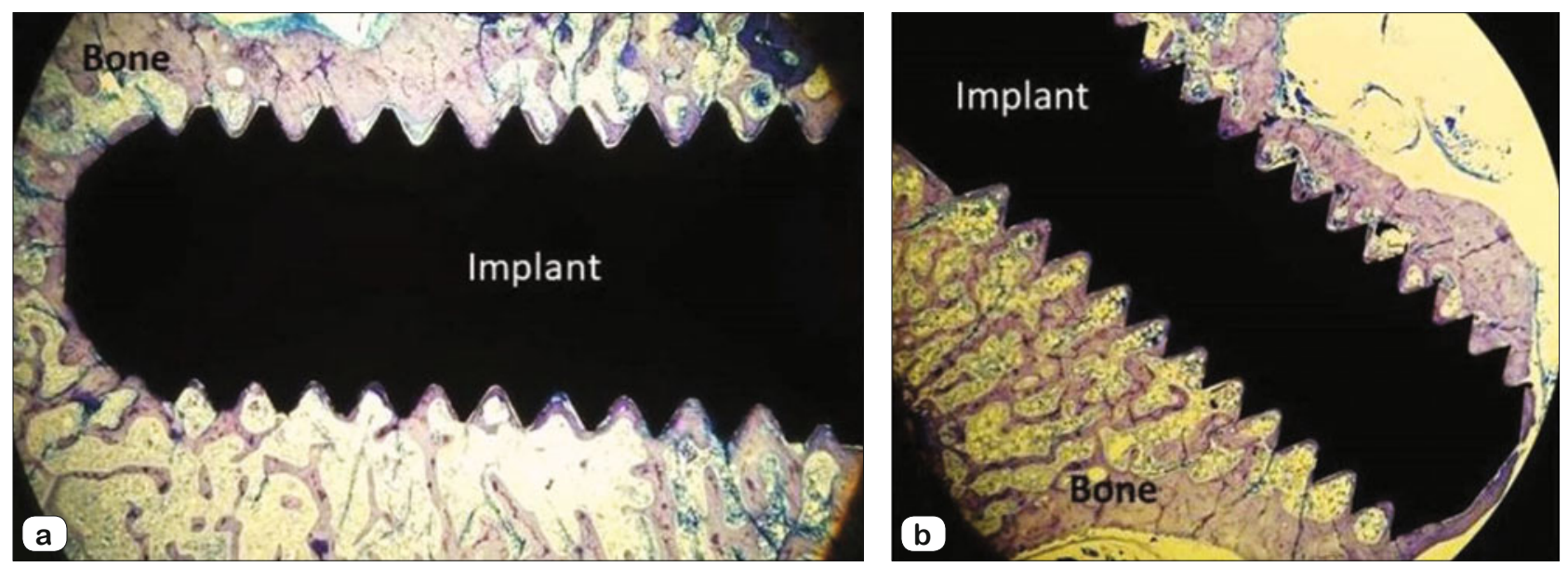

Fig. 1 a, b: Non decalcified histological section of the ND and HFD groups (Toluidin Blue staining, 2X magnification).
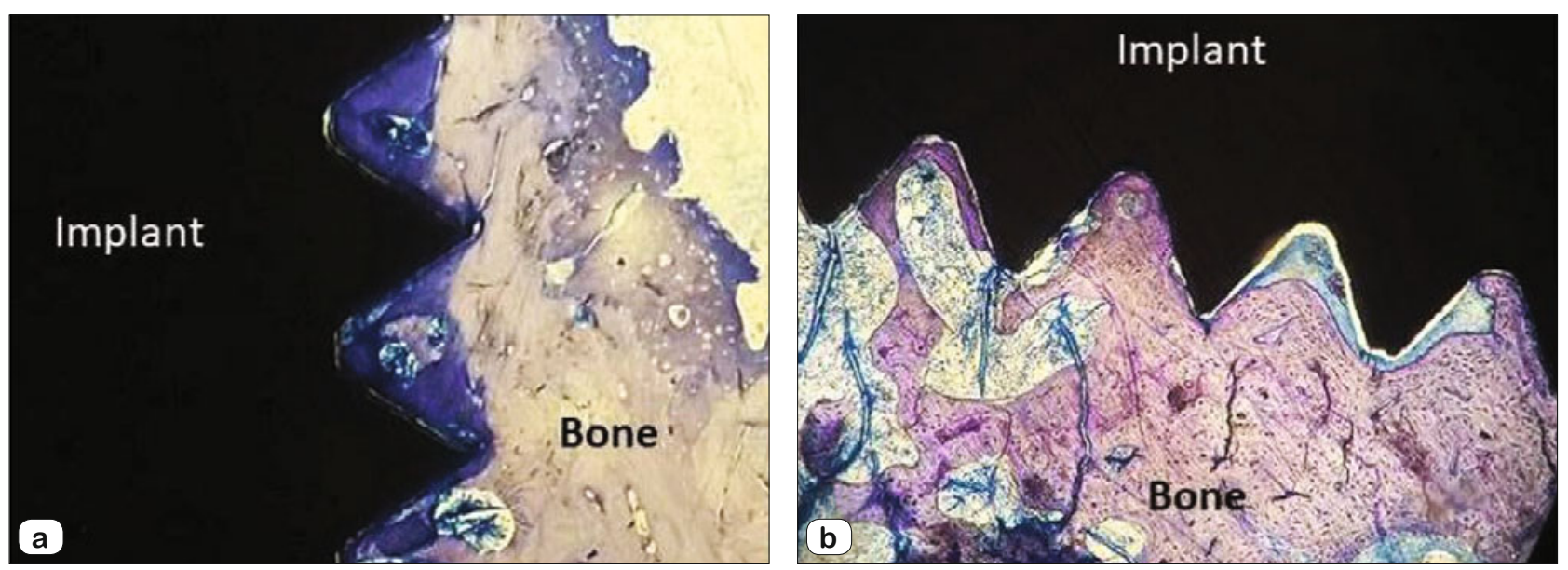

Fig. 2 a, b: Non decalcified histological section of the ND and HFD groups (Toluidin Blue staining, 4X magnification).

$(p=0.001)$ levels were detected to be statistically significantly higher in HFD group compared with the ND group $(\mathrm{p}<0.05)$. Additionally, body weight measurements did not shown statistically significant differences between the two groups at the end of the study (Tab. 2) $(\mathrm{p}>0.05)$.

\section{Dual energy $X$-ray absorptiometry results}

Bone mineral density and BMC data differences were not statistically significant when comparing the ND and HFD groups (p>0.05) (Tab. 2).

\section{Discussion}

The purpose of this study was to examine the effects of HFD before and after the surgical integration of the titanium implants in rat tibias. The non-decalcified histological results in this research did not confirm the hypothesis about the inverse relation between HFD and osseointegration. In the high-fat diet, $42 \%$ of the calorie content was fat. In this research, we preferred Sprague Dawley healthy rats according to the Sahin et. al study (10).
Body weight increase could be negatively related to bone mass because of the possible competitive effect between adipocyte differentiation and osteoblast differentiation, as they share a common progenitor. Fat accumulation is not protective against bone loss. Some studies have reported low osteoblast function and suppressed osteoblastic gene expression in HFD mice, potentially as a consequence of a mesenchymal stem cells differentiation switch favoring adipogenesis. Adipose tissue is not an inert organ with a sole function of storing energy; it has metabolic functions, secreting proteins that are involved in bone metabolism. Indeed, HFD has a greater impact on bone metabolism by endocrine stimulus than by mechanical stimulus, regardless of weight (11-13). Several mechanisms have been proposed to explain HFD-induced osteoclastogenesis, including elevated levels of the pro-inflammatory cytokines IL-1 and TNF in the blood derived from adipose tissue macrophages, increased RANKL expression from bones, and decreased expression of the anti-osteoclastogenic cytokine IL-10 (14-16).

The oxidation factor of lipid and lipid-bound protein molecules in osteoporosis pathophysiology has been reported in various studies. In a previous study, researchers reported that the mineral 
Tab. 2. Weight measurements. biochemical and DEXA parameters of the groups after the $6^{\text {th }}$ month-at the end of the study.

\begin{tabular}{|c|c|c|c|c|c|}
\hline Parameters & Group & $\mathrm{n}$ & Mean & SD & $\mathrm{p}$ \\
\hline \multirow{2}{*}{ Weights (gr) } & HFD & 10 & 292.50 & 42.29 & \multirow[t]{2}{*}{$>0.05$} \\
\hline & ND & 10 & 284.95 & 35.39 & \\
\hline \multirow{2}{*}{ Glucose (mg/dl) } & HFD & 10 & 86.30 & 15.59 & \multirow[t]{2}{*}{$>0.05$} \\
\hline & NF & 10 & 91.60 & 12.49 & \\
\hline \multirow{2}{*}{$\mathrm{LDL}(\mathrm{mg} / \mathrm{dl})$} & HFD & 10 & 2.94 & 0.48 & \multirow[t]{2}{*}{$<0.05(\mathrm{p}=0.002)$} \\
\hline & ND & 10 & 3.72 & 0.48 & \\
\hline \multirow{2}{*}{ VLDL (mg/dl) } & HFD & 10 & 18.46 & 5.19 & \multirow[t]{2}{*}{$<0.05(\mathrm{p}=0.001)$} \\
\hline & ND & 10 & 10.34 & 3.09 & \\
\hline \multirow{2}{*}{ Triglycerids (mg/dl) } & HFD & 10 & 92.30 & 25.95 & \multirow[t]{2}{*}{$<0.05(\mathrm{p}=0.001)$} \\
\hline & ND & 10 & 51.70 & 15.46 & \\
\hline \multirow{2}{*}{ AST (U/L) } & HFD & 10 & 147.40 & 20.33 & \multirow[t]{2}{*}{$>0.05$} \\
\hline & ND & 10 & 140.00 & 26.95 & \\
\hline \multirow{2}{*}{$\operatorname{ALT}(\mathrm{U} / \mathrm{L})$} & HFD & 10 & 44.60 & 7.21 & \multirow[t]{2}{*}{$>0.05$} \\
\hline & ND & 10 & 43.30 & 6.15 & \\
\hline \multirow{2}{*}{$\mathrm{BMD}\left(\mathrm{gr} / \mathrm{cm}^{2}\right)$} & HFD & 10 & 0.3423 & 0.027 & \multirow[t]{2}{*}{$>0.05$} \\
\hline & ND & 8 & 0.3485 & 0.022 & \\
\hline \multirow{2}{*}{$\mathrm{BMC}(\mathrm{gr})$} & HFD & 10 & 0.6280 & 0.051 & \multirow[t]{2}{*}{$>0.05$} \\
\hline & ND & 8 & 0.6250 & 0.038 & \\
\hline \multirow{2}{*}{$\mathrm{P}(\mathrm{mg} / \mathrm{dl})$} & HFD & 9 & 4.90 & 0.71 & \multirow[t]{2}{*}{$>0.05$} \\
\hline & ND & 10 & 5.50 & 1.18 & \\
\hline \multirow{2}{*}{ ALP (IU/L) } & HFD & 10 & 41.2 & 25.71 & \multirow[t]{2}{*}{$>0.05$} \\
\hline & ND & 10 & 56.9 & 57.33 & \\
\hline \multirow{2}{*}{$\mathrm{Ca}(\mathrm{mg} / \mathrm{dl})$} & HFD & 10 & 10.12 & 2.51 & \multirow[t]{2}{*}{$>0.05$} \\
\hline & ND & 10 & 10.60 & 1.93 & \\
\hline
\end{tabular}

content and Bone Volume/Trabecular Volume ratios of both femoral and tibial bones of mice significantly decreased, as well as hyperlipidemic rats fed atherogenic HFD (17-20). In other previous study, the authors reported that HFD-induced obesity during growth not only triggers mandibular osteoporosis but also increases the risk of spontaneous periodontal disease (9). In a study conducted by Lac et al, it was found that mice fed during the early development period with a high-fat diet had lower bone mineral content and bone mineral density (20). In addition, it has been reported that there is a negative correlation between visceral fat ratio and bone mineral density. Moreover $\mathrm{Lu}$ et al. reported similar results in an in vivo study in young male rats (21). Lu et al reported that the HFD feeding significantly decreased the bone mineral content and the trabecular bone area compared with the normal diet feeded rats (21). A low bone density has been reported in atherosclerotic mice.

Pirih et. al. reported that oxidative lipids and/or hyperlipidemia negatively affected the mechanical strength of the bone and disrupted the regeneration process of the bone in the study they performed (22). Additionally, they reported that in HFD group cortical bone volume fraction was significantly decreased in femoral bone when compared with the normal diet rats. Hyperlipidemia affects bone healing negatively, thus reducing bone surface and volume. In this study, LDL, VLDL and triglyceride levels in the subjects fed with HFD were found to be statistically higher than in controls, but glucose, cholesterol, HDL, liver parameters and weight measurement (AST, ALT) did not differ between the two groups. These results may be indicative of the effect of HFD on blood fat metabolism.
It has previously been reported that oxidized lipids inhibit the differentiation of osteoblasts and increase the apoptosis of osteoblasts (23). In addition, epidemiological studies report that osteoporosis may be associated with hyperlipidemia and atherosclerosis (24). The result obtained was independent of age for some populations (25). Keuroghlian et al. reported that atherosclerosis susceptible C57BL/6J male mice fed a high-fat diet reduced the amount and strength of bone-implant contact in the femur bones and significantly increased implant loss (26). These results support the hypothesis that HFD may reduce osseointegration and lead to adverse outcomes in dental implant therapy. Our histological and histomorphometric results do not support the results reported by Keuroglian et al (26). Dundar et al. reported that, the BIC ratio in rabbits fed the 12-week HFD diet did not show any difference compared to the rabbits fed for 12 weeks normal diet (6). In this study, the BIC ratio in the HFD group did not show a statistically significant difference when compared to the controls. Keuroglian et al. reported that more than one mechanism is effective in reducing bone-implant contact in HFD-fed rats (26). Cell-level hyperlipidemic conditions lead to inhibition of osteogenic signaling, reduction of mature osteoblast formation, higher production of molecular markers in bone remodeling, enhancement of osteoclast differentiation and activity, and increase of bone resorption.

Testing oseointegration of the titanium implants in rat tibial bones has some limitations due to differences in bone-implant interaction, microstructure, composition and bone remodeling. Long bones have less bone remodeling capacity due to a larger bone marrow space when compared with the jaws. In addition, in this study, the implants were not loaded, which is another limitation. Also, rats used in this study were not atherosclerosis-susceptible rats (26).

\section{Conclusion}

In this study, we fed rats 3 months before implant integration and 3 months after implant integration. Within the limitation of this study, we concluded that HFD could not decrease the BIC of the implants in rat tibial bone model. However, further studies are needed to clarify the relationship between the BIC and HFD.

\section{References}

1. Frisch E, Ziebolz D, Rinke S. Long-term results of implant supported over-denture retained by double crowns: a practice based retrospective study after minimally 10 years follow-up. Clin Oral Implants Res 2013; 24 (12): 1281-1287.

2. Dundar S, Yaman F, Bozoglan A, Yildirim TT, Kirtay M, Ozupek MF et al. Comparison of Osseointegration of Five Different Surfaced Titanium Implants. J Craniofac Surg 2018; 29 (7): 1991-1995.

3. Pirih F, Lu J, Ye F, Bezouglaia O, Atti E, Ascenzi MG et al. Adverse effects of hyperlipidemia on bone regeneration and strength. J Bone Miner Res 2012; 27: 309-318.

4. Tintut Y, Morony S, Demer LL. Hyperlipidemia promotes osteoclastic potential of bone marrow cells ex vivo. Arterioscler Thromb Vasc Biol 2004; 24: e6-10. 
$450-454$

5. Brodeur MR, Brissette L, Falstrault L, Ouellet P, Moreau R. Influence of oxidized low-density lipoproteins (LDL) on the viability of osteoblastic cells. Free Radic Biol Med 2008; 44: 506-517.

6. Dündar S, Yaman F, Ozupek MF, Saybak A, Gul M, Asutay F et al. The effects of high-fat diet on implant osseointegration: an experimental study. J Korean Assoc Oral Maxillofac Surg 2016; 42 (4): 187-192.

7. Wohl GR, Loehrke L, Watkins BA, Zernicke RF. Effects of high-fat diet on mature bone mineral content, structure, and mechanical properties. Calcif Tissue Int 1998; 63 (1): 74-79.

8. Verzeletti GN, Gaio EJ, Linhares DS, Rosing CK. Effect of obesity on alveolar bone loss in experimental periodontitis in Wistar rats. J Appl Oral Sci 2012; 20 (2): 218-221.

9. Fujita Y, Maki K. High-fat diet-induced obesity triggers alveolar bone loss and spontaneous periodontal disease in growing mice. BMC Obes 2016 8; 3: 1. doi: 10.1186/s40608-016-0082-8. eCollection 2015.

10. Sahin K, Tuzcu M, Orhan C, Agca CA, Sahin N, Guvenc M et al. The effects of chromium complex and level on glucose metabolism and memory acquisition in rats fed high-fat diet. Biol Trace Elem Res 2011; 143 (2): 1018-1030.

11. Yamanaka JS, Yanagihara GR, Carlos BL, Ramos J, Brancaleon BB, Macedo AP et. al. A high-fat diet can affect bone healing in growing rats. J Bone Miner Metab 2018; 36 (3): 255-263

12. Vendrell J, Broch M, Vilarrasa N, Molina A, Gómez JM. Adiponectin, ghrelin, leptin, and proinflammatory cytokines: relationships in obesity. Obes Res 2004; 12: 962-971.

13. Greco EA, Lenzi A, Migliaccio S. The obesity of bone. Ther Adv Endocrinol Metab 2015; 6: 273-286.

14. Shu L, Beier E, Sheu T, Zhang H, Zuscik MJ, Puzas EJ et al. Highfat diet causes bone loss in young mice by promoting osteoclastogenesis through alteration of the bone marrow environment. Calcif Tissue Int 2015; 96 (4): 313-323.

15. Kyung TW, Lee JE, Phan TV, Yu R, Choi HS. Osteoclastogenesis by bone marrow-derived macrophages is enhanced in obese mice. J Nutrition 2009; 139: 502-506.
16. Woo DG, Lee BY, Lim D, Kim HS. Relationship between nutrition factors and osteopenia: effects of experimental diets on immature bone quality. J Biomech 2009; 42: 1102-1107

17. Graham LS, Tintut Y, Parhami F, Kitchen CM, Ivanov Y, Tetradis S, et al. Bone density and hyperlipidemia: the T-lymphocyte connection. J Bone Miner Res 2010; 25: 2460-2469.

18. Parhami F. Possible role of oxidized lipids in osteoporosis: could hyperlipidemia be a risk factor? Prostaglandins Leukot Essent Fatty Acids 2003; 68: 373-378.

19. Rajamannan NM. Low-density lipoprotein and aortic stenosis. Heart 2008; 94: 1111-1112.

20. Lac G, Cavalie H, Ebal E, Michaux O. Effects of a high fat diet on bone of growing rats. Correlations between visceral fat, adiponectin and bone mass density. Lipids Health Dis 2008; 7: 16.

21. Lu XM, Zhao H, Wang EH. A high-fat diet induces obesity and impairs bone acquisition in young male mice. Mol Med Rep 2013; 7: 1203-1208.

22. Pirih F, Lu J, Ye F, Bezouglaia O, Atti E, Ascenzi MG et al. Adverse effects of hyperlipidemia on bone regeneration and strength. J Bone Miner Res 2012; 27: 309-318.

23. Hirasawa H, Tanaka S, Sakai A, Tsutsui M, Shimokawa H, Miyata H et al. ApoE gene deficiency enhances the reduction of bone formation induced by a high-fat diet through the stimulation of p53-mediated apoptosis in osteoblastic cells. J Bone Miner Res 2007; 22: 1020-1030.

24. Boukhris R, Becker KL. Calcification of the aorta and osteoporosis. A roentgenographic study. JAMA 1972; 219: 1307-1311.

25. Jie KG, Bots ML, Vermeer C, Witteman JC, Grobbee DE. Vitamin $\mathrm{K}$ status and bone mass in women with and without aortic atherosclerosis: a population-based study. Calcif Tissue Int 1996; 59: 352-356.

26. Keuroghlian A, Barroso AD, Kirikian G, Bezouglaia O, Tintut Y, Tetradis $\mathbf{S}$, et al. The effects of hyperlipidemia on implant osseointegration in the mouse femur. J Oral Implantol 2015; 41: e7-11.

Received February 12, 2019. Accepted February 19, 2020. 